Article

\title{
Closed-Loop Control of Droplet Transfer in Electron-Beam Freeform Fabrication
}

\author{
Shuhe Chang ${ }^{1,2}$, Haoyu Zhang ${ }^{1}$, Haiying $X u^{3}$, Xinghua Sang ${ }^{3}$, Li Wang ${ }^{1}$, Dong Du ${ }^{2, *}$ and \\ Baohua Chang ${ }^{1, *}$ \\ 1 Department of Mechanical Engineering, Tsinghua University, Beijing 100084, China; \\ changsh15@mails.tsinghua.edu.cn (S.C.); z-hy18@mails.tsinghua.edu.cn (H.Z.); \\ wanglidme@mail.tsinghua.edu.cn (L.W.) \\ 2 Key Laboratory for Advanced Materials Processing Technology, Ministry of Education, Tsinghua University, \\ Beijing 100084, China \\ 3 Manufacturing Technology Institute, Aviation Industry Corporation of China, Beijing 100024, China; \\ xhyxhy@126.com (H.X.); sang_xh@163.com (X.S.) \\ * Correspondence: dudong@tsinghua.edu.cn (D.D.); bhchang@tsinghua.edu.cn (B.C.)
}

Received: 19 January 2020; Accepted: 6 February 2020; Published: 10 February 2020

\begin{abstract}
In the process of electron-beam freeform fabrication deposition, the surface of the deposit layer becomes rough because of the instability of the feeding wire and the changing of the thermal diffusion condition. This will make the droplet transfer distance change in the deposition process, and the droplet transfer cannot always be stable in the liquid bridge transfer state. It is easy to form a large droplet or make wire and substrate stick together, which makes the deposition quality worsen or even interrupts the deposition process. The current electron-beam freeform fabrication deposition is mostly open-loop control, so it is urgent to realize the real-time and closed-loop control of the droplet transfer and to make it stable in the liquid bridge transfer state. In this paper, a real-time monitoring method based on machine vision is proposed for the droplet transfer of electron-beam freeform fabrication. The detection accuracy is up to $\pm 0.08 \mathrm{~mm}$. Based on this method, the measured droplet transfer distance is fed back to the platform control system in real time. This closed-loop control system can stabilize the droplet transfer distance within $\pm 0.14 \mathrm{~mm}$. In order to improve the detection stability of the whole system, a droplet transfer detection algorithm suitable for this scenario has been written, which improves the adaptability of the droplet transfer distance detection method by means of dilatation/erosion, local minimum value suppression, and image segmentation. This algorithm can resist multiple disturbances, such as spatter, large droplet occlusion and so on.
\end{abstract}

Keywords: metal additive manufacturing; electron-beam freeform fabrication; droplet transfer; closed-loop control

\section{Introduction}

In recent years, the technology of metal additive manufacturing has been developed rapidly, attracting the attention of many researchers. There are many ways to additively manufacture metals, including Electron Beam Melting (EBM), Selective Laser Melting (SLM), Direct Metal Laser Sintering (DMLS), Hot Wire Gas Tungsten Arc Welding (HW-GTAW), and Ion Fusion Formation (IFF). The most remarkable feature of additive manufacturing is that a product is made layer-by-layer without mold or tooling. The computer aided design (CAD) model is divided into layers of consistent thickness by slicing software. Only one layer is deposited at a time, and the layers are stacked layer-by-layer to form the final part [1,2]. Electron-Beam Freeform Fabrication (EBF3) is one of the metal additive manufacturing technologies. The processed parts can reach the strength of forgings. Compared with 
other metal additive manufacturing methods, EBF3 can achieve a higher deposition efficiency with quality assurance because the vacuum environment is beneficial to the protection of parts [3,4].

The instability of the droplet transfer will, however, cause the quality of the electron-beam freeform fabrication to worsen or even cause the interruption of the deposition process [5-7]. It is urgent to realize the closed-loop control of the droplet transfer, which will make the deposition process stable in the liquid bridge transfer state, but the rough surface of the deposited layer will cause instability of the droplet transfer [8-12]. Geometric errors occur because of the instability of the heat input and change in the thermal diffusion conditions. The surface of the deposition layer will become obviously uneven after layer-by-layer accumulation, even if the geometric error is very small in each layer. The height of the wire feeder is usually fixed during the EBF3, so when the surface of the deposited layer is higher than the target surface, the droplet transfer distance decreases, and the wire and substrate become easier to adhere to one another, resulting in the interruption of the deposition process. Further, when the surface of the deposited layer is concave, the droplet transfer distance increases, which results in a large droplet at this time and seriously affects the forming quality [5]. In order to ensure the deposition quality, it is urgent to control the droplet transfer distance in real time so that the deposition process is stable in the transfer state of the liquid bridge. In order for electron-beam freeform fabrication technology to be applied in the environment of space, a stable liquid bridge transfer must also be realized. When deposited on Earth, the droplets are still able to transfer by gravity. In the environment of space, however, weightlessness makes it impossible to rely on gravity for droplet transfer-only surface tension can be relied on for droplet transfer [13]. This requires the deposition process to maintain a very stable liquid bridge transfer.

Optical images are often used for real-time closed-loop control because of their adequate information. Heralić et al. [14,15] detected the height of the deposition layer offline using optical methods, but could not achieve real-time control. Zeng et al. [16-18] proposed a welding pass-detection method based on directional light and structure-light information fusion, aiming to tackle the problem that the structure-light laser could not obtain the welding pass information stably under the condition of strong mirror reflection. However, in the process of EBF3, the lens in front of the laser will be coated with a layer of metal vapor which makes the light transmittance drop sharply and, therefore, not meet the need of long-time stable work. Chang et al. $[19,20]$ realized the 3D reconstruction of the electron-beam freeform fabrication process based on electron-beam structured-light technology instead of laser-structured light. However, it must be scanned during the deposition interval and it is impossible to calculate the height of the deposited layer in real time during the deposition process. So the real-time control of the droplet transfer cannot be realized. Taminger et al. [7] proposed a closed-loop EBF3 control method where a side-view optical camera can be used to monitor the height of a deposited bead on substrate. The Z-height of the deposit was adjusted up or down to maintain a consistent deposition distance and eliminate wire sticks and drips associated with incorrect standoff distance between the wire feeder and the deposit. However, this patent only gives the original idea, instead of the specific image-processing algorithm, as well as the realized detection accuracy and control accuracy.

In this paper, a vision-based method is proposed to monitor the droplet transfer distance and adjust the substrate height in real time to achieve a stable liquid bridge transfer.

\section{Effect of Droplet Transfer Distance on Deposition Quality}

\subsection{Definition of Droplet Transfer Distance}

A schematic of a typical droplet transfer is shown in Figure 1. The edge line of the electron beam near the side of the wire is denoted as $\mathrm{L}_{0}$. The long axis of the molten pool is denoted as $\mathrm{L}_{1}$. The central axis of the wire is denoted as $\mathrm{L}_{2}$. The intersection of $\mathrm{L}_{0}$ and $\mathrm{L}_{1}$ is denoted as $\mathrm{P}_{1}$. The intersection of $\mathrm{L}_{0}$ and $L_{2}$ is denoted as $P_{2}$. The distance between $P_{1}$ and $P_{2}$ is defined as $\Delta h$, which represents the droplet transfer distance. 


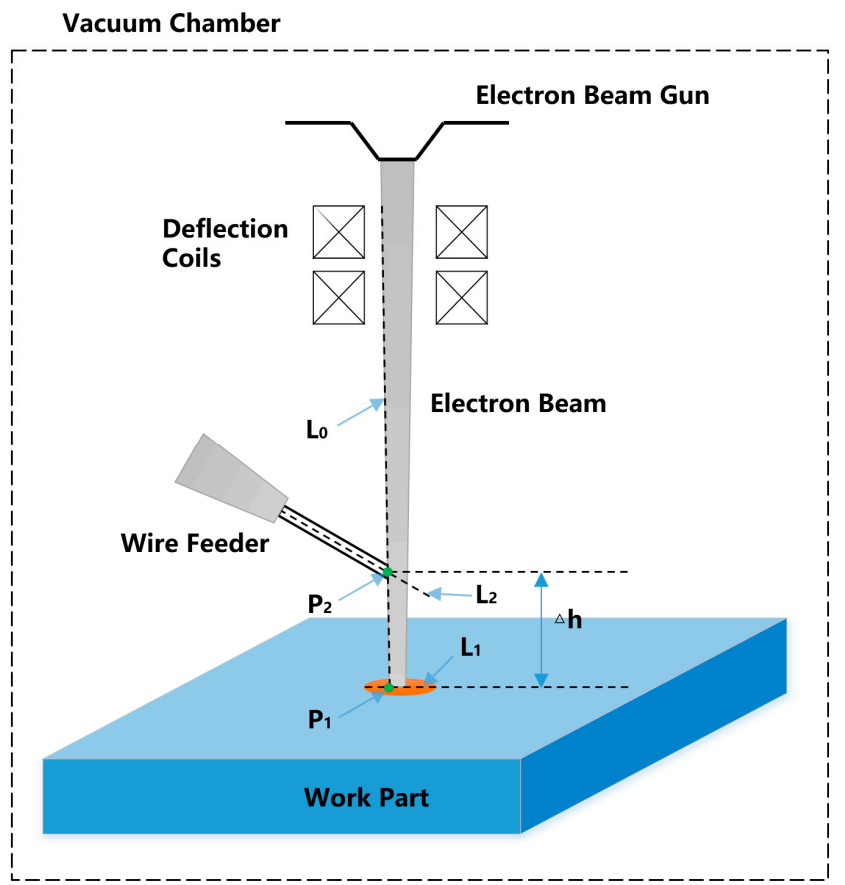

Figure 1. Definition of droplet transfer distance.

2.2. Different Droplet Transfer States Under Different Droplet Transfer Distances

A $50 \mathrm{~mm}$ line deposit is produced with the droplet transfer distance changed linearly from $0 \mathrm{~mm}$ to $5 \mathrm{~mm}$. The experimental parameters are shown in Table 1.

Table 1. Experimental parameters.

\begin{tabular}{ccc}
\hline Parameter & Value & Unit \\
\hline Acceleration voltage & 50 & $\mathrm{kV}$ \\
Electron-beam current & 50 & $\mathrm{~mA}$ \\
Deposition speed & 400 & $\mathrm{~mm} / \mathrm{min}$ \\
Wire-feed speed & 1.5 & $\mathrm{~m} / \mathrm{min}$ \\
Droplet transfer distance & $0 \sim 5$ & $\mathrm{~mm}$ \\
Deposition length & 50 & $\mathrm{~mm}$ \\
\hline
\end{tabular}

The droplet transfer states under different droplet transfer distances are shown in Figure 2. It can be seen that in $0 \sim 1 \mathrm{~mm}$, the droplet transfer presents a liquid bridge transfer form. When the droplet transfer distance is $1 \sim 5 \mathrm{~mm}$, large droplet transfer begins to appear, and the droplet diameter gradually increases.

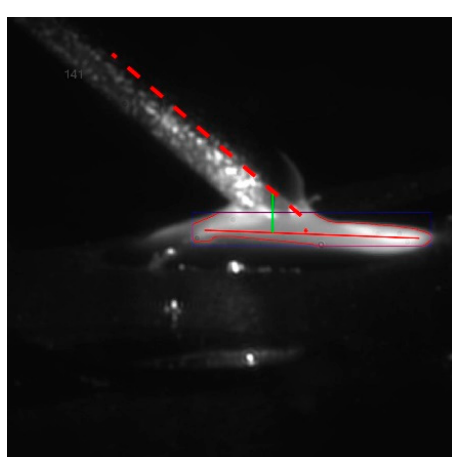

$0 \mathrm{~mm}$

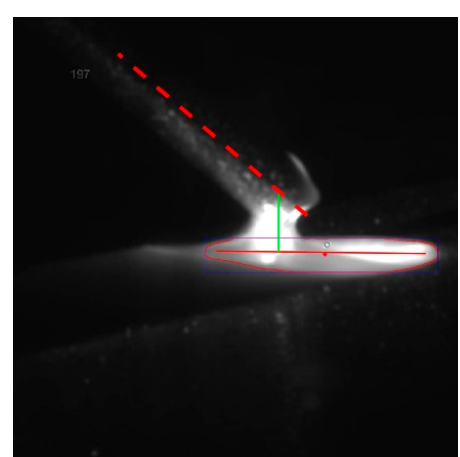

$1 \mathrm{~mm}$

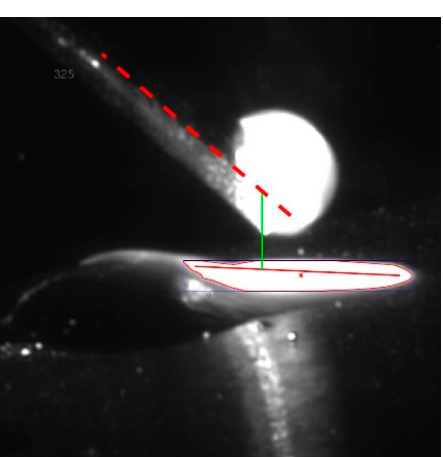

$2 \mathrm{~mm}$

Figure 2. Cont. 


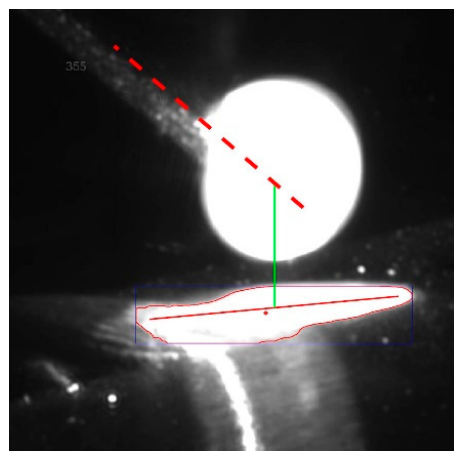

$3 \mathrm{~mm}$

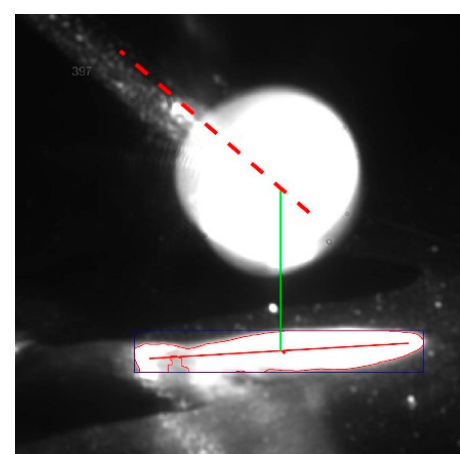

$4 \mathrm{~mm}$

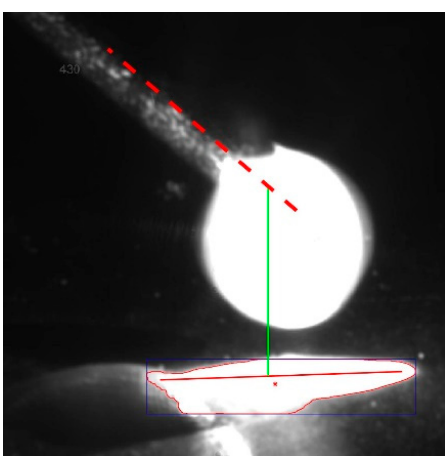

$5 \mathrm{~mm}$

Figure 2. Different droplet transfer states under different droplet transfer distances.

\section{Image Processing Algorithm for Calculation of Droplet Transfer Distance}

As mentioned in the previous section, in order to obtain the droplet transfer distance, it is necessary to obtain the position of the intersection point $P_{1}$ between the long axis of the molten pool and the sideline of the electron beam, in order to calculate the distance between $\mathrm{P}_{1}$ and $\mathrm{P}_{2}$. The drop transfer images obtained by a camera usually have noise points; at the same time, there will be interference factors such as big droplet occlusion and miscellaneous-light reflection in the process of deposition. Hence, the methods of image processing are required to obtain the droplet transfer distance quickly and stably. The entire image processing flow proposed in this study is shown in Figure 3a.

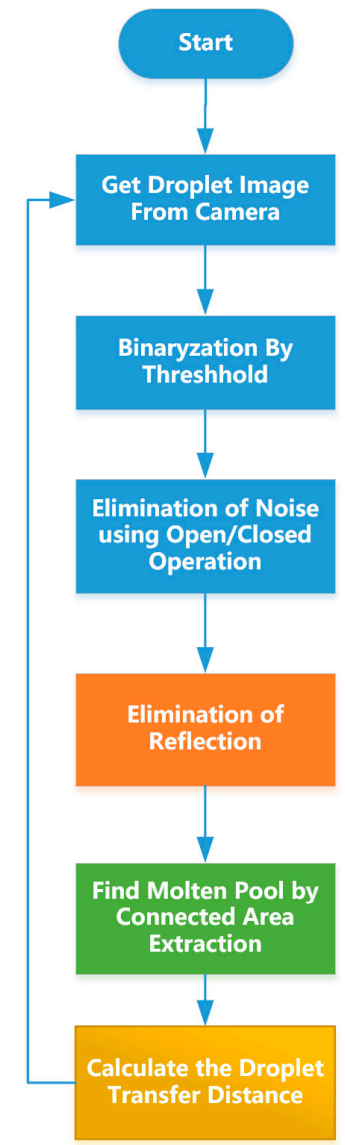

(a)

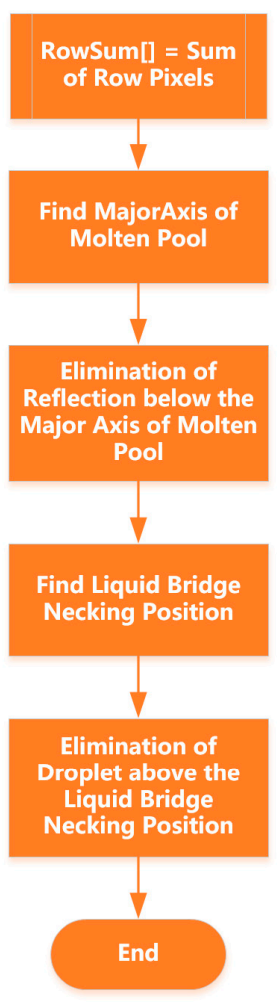

(b)

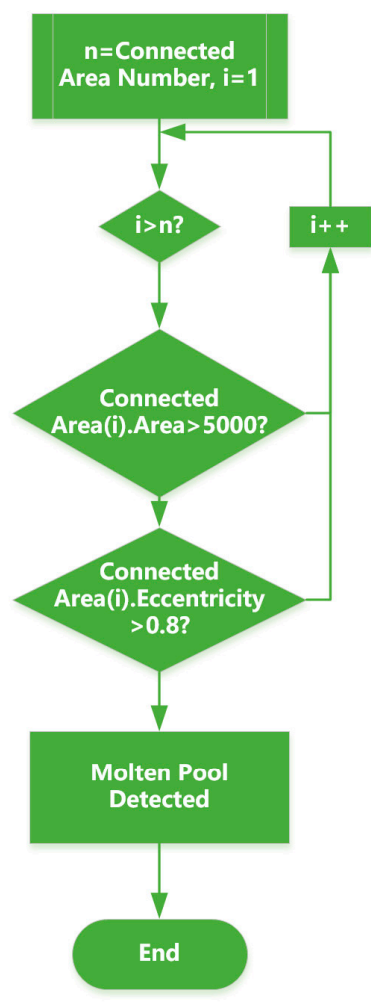

(c)

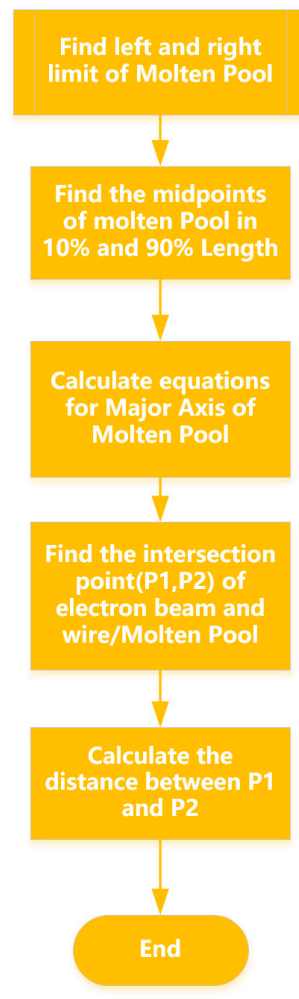

(d)

Figure 3. Flowchart of image processing: (a) main program of image processing algorithm; (b) subroutine for image segmentation; (c) subroutine for molten-pool extraction; (d) subroutine for calculation of droplet transfer distance. 


\subsection{Image Preprocessing}

The droplet transfer image is captured by an industrial camera, as shown in Figure 4a. Firstly, the original image is binarized on a preset threshold to facilitate the extraction of the molten pool. The binarized droplet transfer image is shown in Figure $4 \mathrm{~b}$. It can be seen from Figure $4 \mathrm{~b}$ that after being binarized, some miscellaneous points and solidification areas around the lower grey value have been eliminated. There are still some high-level noise points in the figure due to spatter and reflection. The image is first eroded and then dilated by a square connected domain with a size of 20. The droplet transfer image after erosion/dilatation is shown in Figure 4c. As we can see from the figure, the spatter and small reflection area can be eliminated.

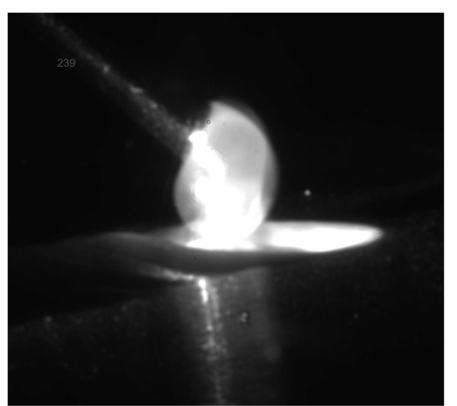

(a)

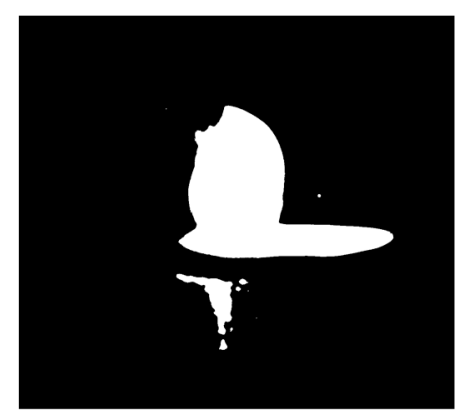

(b)

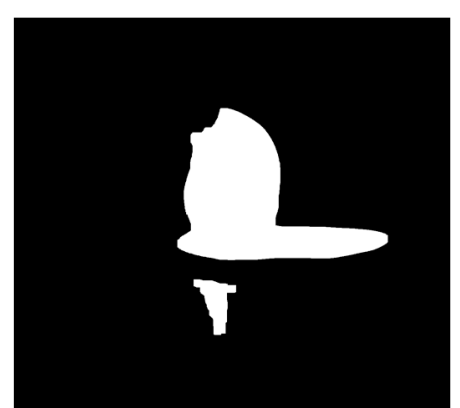

(c)

Figure 4. Image sequence of image preprocessing: (a) original droplet transfer image; (b) image after binarization; (c) image after erosion/dilatation.

\subsection{Image Segmentation}

The preprocessed images are divided into two regions: the droplet and the molten pool overlapping regions and the reflection regions. In order to accurately calculate the long axis of the molten pool, it is necessary to segment the image and accurately separate the droplet, the molten pool and reflection area. The flow chart is shown in Figure $3 b, c$. The image is accumulated along the row according to Equation (1), and the pixel accumulation distribution curve $\left(\mathrm{Y}_{i}\right)$ along the image row is obtained, as shown in Figure 5a. First, the maximum peak point of the whole curve is found, and the row index $\left(i_{\text {max }}\right)$ of this point is regarded as the height value at the maximum length of the molten pool. Then, it is necessary to find the local minimum point of the curve $\left(\mathrm{Y}_{i}\right)$ from $i=\left(i_{\text {max }}-200\right)$ to $i=i_{\text {max }}$. The row index $\left(i_{\text {droplet }}\right)$ of this point is considered the height of the horizontal boundary between the molten pool and the droplet. Afterward, it is necessary to find the local minimum point of the curve $\left(\mathrm{Y}_{i}\right)$ from $i=i_{\text {max }}$ to $i$ $=\left(i_{\text {max }}+200\right)$. The row index $\left(i_{\text {reflection }}\right)$ of this point is considered the height of the horizontal boundary between the molten pool and reflection. The segmented image is shown in Figure 5b. The aspect ratio and center of the smallest external rectangle of each region are then calculated, respectively. In the connected domain where the aspect ratio is greater than 4 and the pixel area is greater than 5000, the closest connected domain to the height at the maximum length of the molten pool is considered as the molten pool.

$$
Y_{i}=\sum_{j=1}^{j=1168} I(i, j)
$$

where $i$ is the row index of the image, $j$ is the column index of the image, $I(i, j)$ is the grey value of the pixel in row $\mathrm{i}$, column $\mathrm{j}$ of the image, $Y_{i}$ is the cumulative grey value in row $i$ of the image. 


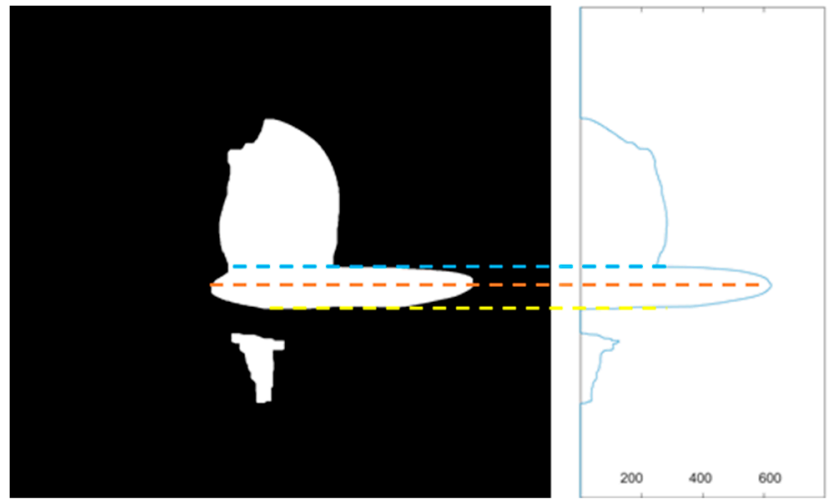

(a)

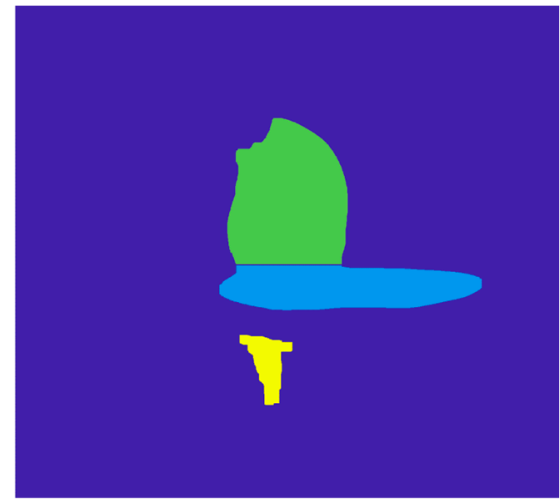

(b)

Figure 5. (a) Accumulation along the image row, where the orange dotted lines indicate the height of the maximum length of the molten pool, the blue dotted lines indicate the height of the boundary between the molten pool and droplet, the yellow dotted lines indicate the height of the boundary between the molten pool and reflection. The horizontal axis represents Yi (the cumulative grey value in row $\mathrm{i}$ of the image). (b) Image segmentation result.

\subsection{Calculation of Droplet Transfer Distance}

After obtaining the molten pool area, it is necessary to extract the long axis $\mathrm{L}_{1}$ of the molten pool in Figure 6a, whose flow chart is shown in Figure $3 \mathrm{~d}$. The left and right limit points $\mathrm{T}_{1}$ and $\mathrm{T}_{2}$ of the molten pool are first extracted, as shown in Figure 6b. To avoid unnecessary fluctuation of the molten pool majoraxis detection caused by the drift of the marginal point, the left and right limit points are moved to $10 \%$ of the maximum molten pool length inside the molten pool. After that, the midpoint $\mathrm{M}_{1}$ and $\mathrm{M}_{2}$ of the upper and lower points of the pool profile is calculated at this horizontal position. The line of $\mathrm{M}_{1}$ and $\mathrm{M}_{2}$ was used as the molten pool long axis $\mathrm{L}_{1}$. Because the position of the wire and electron beam is fixed, the position of them is calibrated in advance of the experiment. Taking the intersection point $\mathrm{P}_{1}$ of $\mathrm{L}_{1}$ and $\mathrm{L}_{0}$, and the intersection point $\mathrm{P}_{2}$ of $\mathrm{L}_{2}$ and $\mathrm{L}_{0}$, the pixel distance of $\mathrm{P}_{1}$ and $\mathrm{P}_{2}$ is calculated as $\Delta \mathrm{h}^{\prime}$, denoting the droplet transfer distance in pixels.

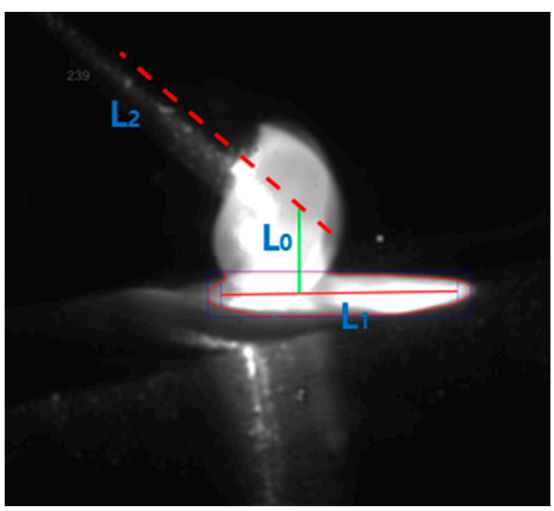

(a)

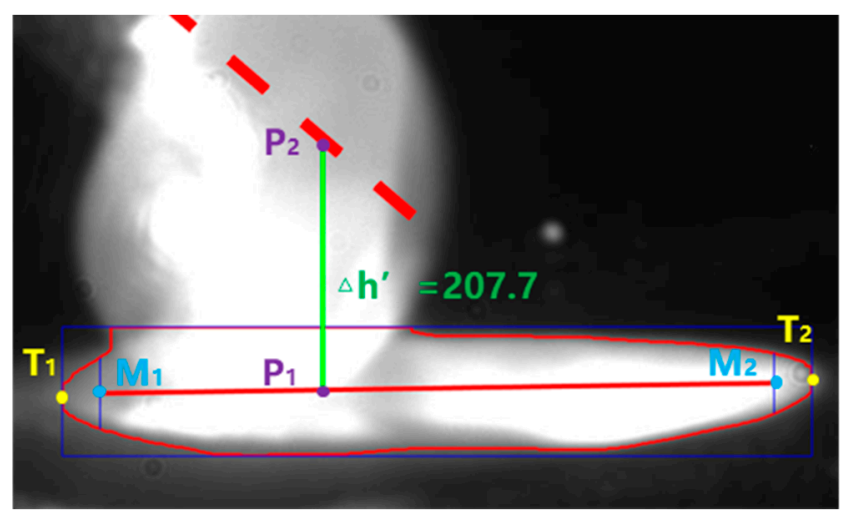

(b)

Figure 6. (a) Advance calibration of $\mathrm{L}_{0}$ and $\mathrm{L}_{2} ;(\mathbf{b})$ calculation of droplet transfer distance in pixels.

\subsection{Transformation From Pixel to Millimeter}

The camera should be calibrated to calculate the distance of the droplet transfer $\Delta \mathrm{h}$ in millimeters from the distance $\Delta \mathrm{h}^{\prime}$ in pixels. The principle of calibration is shown in Figure 7 . After the electron beam is emitted from the cathode of the electron gun, it is vertically incident on the substrate surface, intersecting with the deposition surface at point $\mathrm{P}_{1}$, and intersecting with the wire at point $\mathrm{P}_{2}$. $\mathrm{P}_{1}$ passes through the lens and focuses on point $\mathrm{B}_{1}$ of the CMOS chip. The real distance of the droplet 
transfer is defined as $\Delta \mathrm{h}$, and the pixel distance of the droplet transfer is defined as $\Delta \mathrm{h}$ '. The angle between the electron beam and the imaging light axis is defined as $\alpha$. The focal length of lens is $f$. The object distance is $u 1$ and the image distance is $v 1$. The corresponding relation between the pixel distance $\Delta \mathrm{h}^{\prime}$ of the droplet transfer and the real distance $\Delta \mathrm{h}$ of the droplet transfer can then be obtained by geometric calculation:

$$
\Delta \mathrm{h}=\frac{u 1}{v 1 * \sin \alpha} * \Delta \mathrm{h}^{\prime}
$$

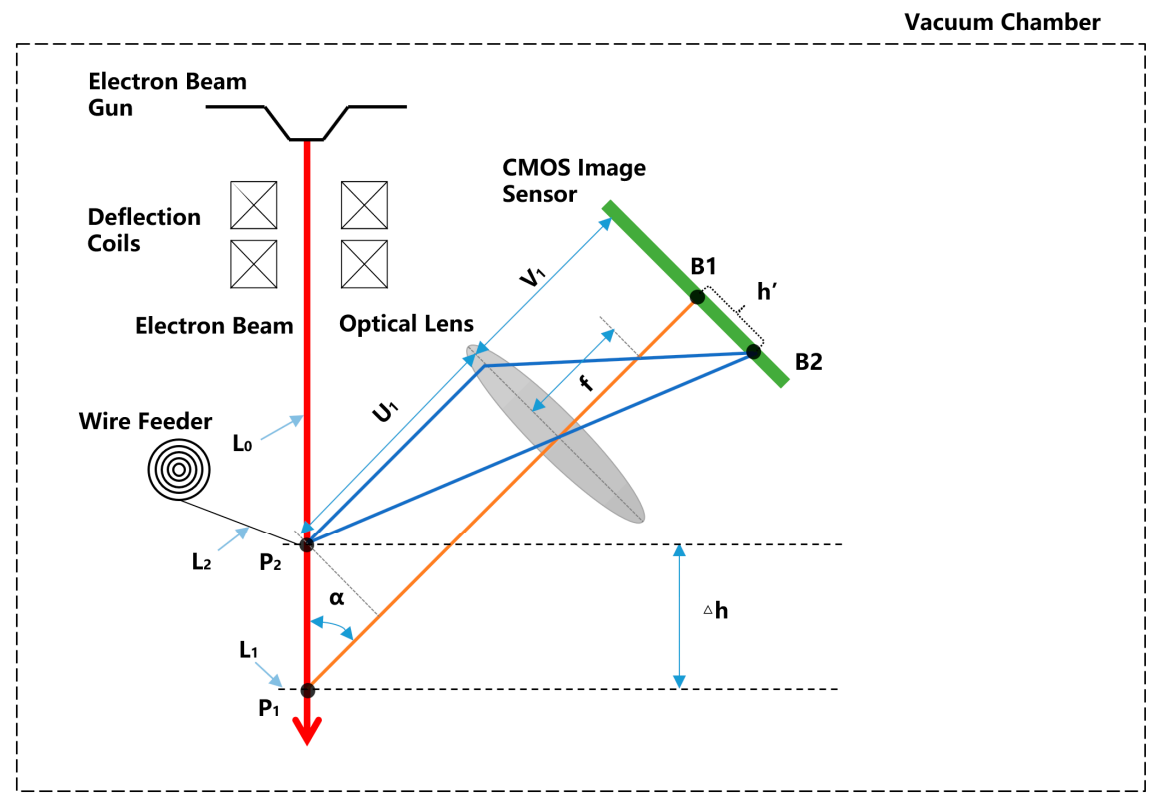

Figure 7. Principle of transformation from pixels to millimeters.

\section{Experiment of Closed-Loop Control of Droplet Transfer}

\subsection{Introduction of Experimental Equipment}

The entire system is schematically shown in Figure 8. The camera used to capture the droplet transfer image sends the image to the industrial control computer through the network cable. The industrial control computer obtains the droplet transfer distance $\Delta \mathrm{h}$ by image processing. The deviation $\mathrm{e}$ is obtained by subtracting the detected droplet transfer distance $\Delta \mathrm{h}$ from the preset-expected droplet transfer distance $\Delta \mathrm{h}_{0}$. In order to realize the closed-loop control of droplet transfer distance, the droplet transfer deviation e is sent to the numeric platform by the OPC-UA communication module of the Siemens 840Dsl CNC system. The 840Dsl CNC system then compensates the droplet transfer distance in real time by adjusting the platform height according to e. The physical system is shown in Figure 9. The system consists of an $15 \mathrm{~kW}$ electron gun for generating an electron beam. The maximum acceleration voltage is $60 \mathrm{kV}$. A three-degrees-of-freedom motion platform for placing the substrate is located in the vacuum chamber. The motion range of the platform is $1000 \mathrm{~mm}$ $(\mathrm{X}) \times 500 \mathrm{~mm}(\mathrm{Y}) \times 500 \mathrm{~mm}(\mathrm{Z})$. An Image Source industrial camera is adopted to acquire the droplet transfer image. The frame rate is $20 \mathrm{fps}$. The CMOS sensor chip size is $1 \mathrm{inch}$, with a resolution of

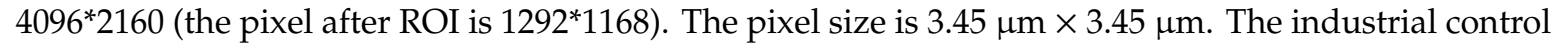
computer is configured with an E5-1650 processor and 32GB memory. 


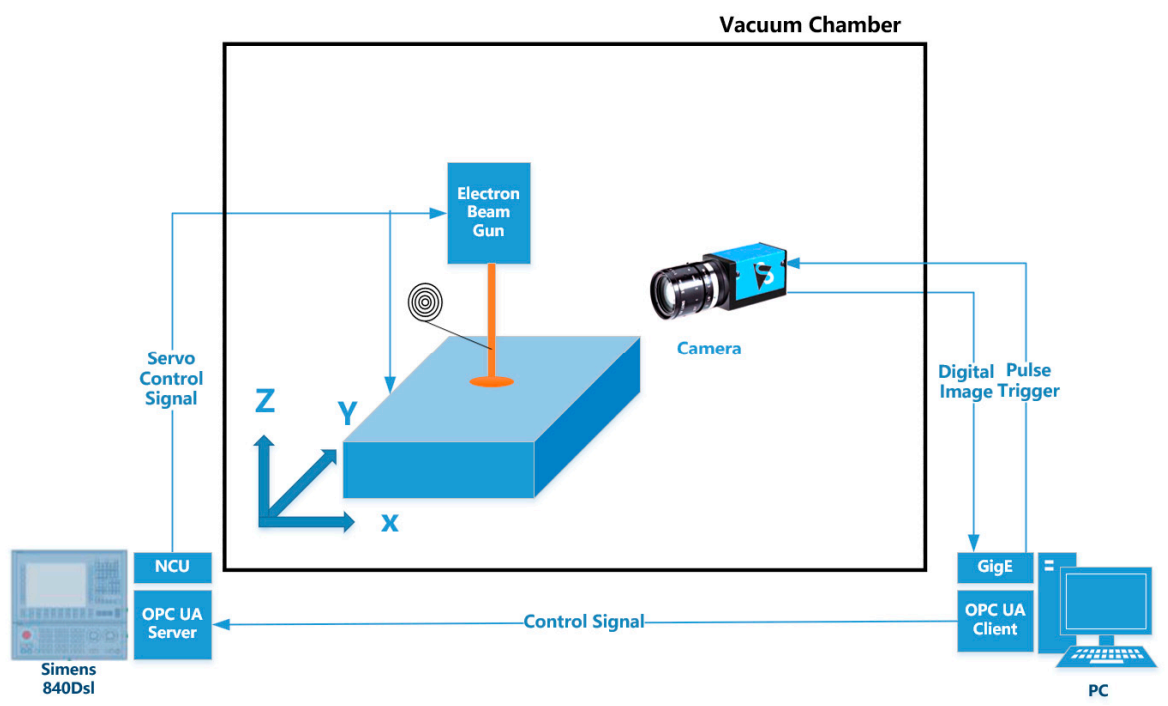

Figure 8. Composition of the closed-loop control system.

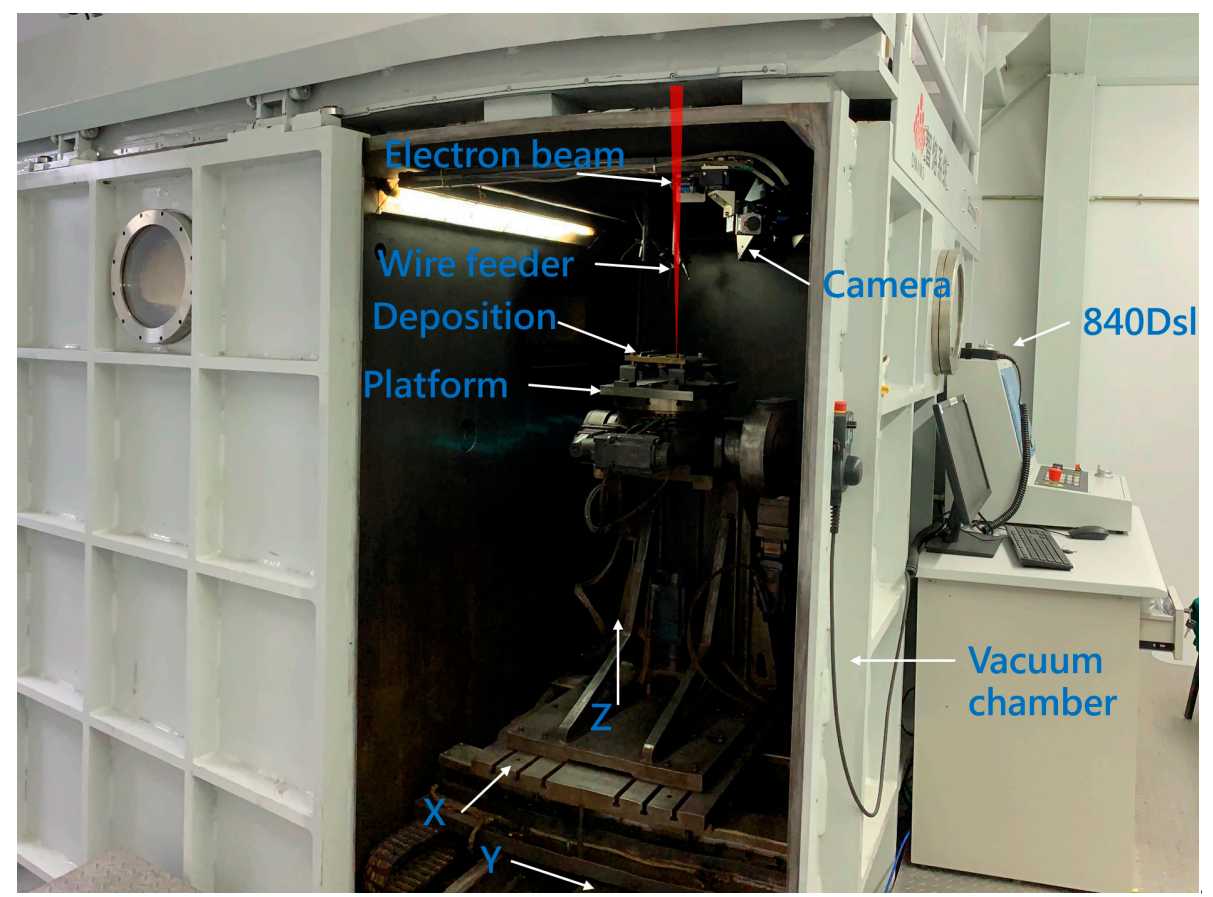

Figure 9. Physical system for the closed-loop control experiment.

\subsection{Flowchart of the Closed-Loop Control System}

The flowchart of the entire system is shown in Figure 10. The electronic gun and the motion platform are controlled by the Siemens 840Dsl numerical control system. The electron beam is accelerated by a high-voltage electric field to melt the wire and substrate to form a droplet transfer. At this point, the IPC generates a signal that triggers the camera to capture the droplet transfer image. The image collected by the camera is transmitted to the industrial control computer through the Gig-e port. The droplet transfer pixel distance is obtained by the image processing program. The real distance of the droplet transfer is calculated according to Equation (2) in the previous section. The droplet transfer distance deviation e is calculated and sent to the controller. The output value of the controller is transmitted to the $840 \mathrm{dsl}$ NC system through the OPCUA communication. Then, the height of the platform is adjusted in real time to make the droplet transfer distance stable. 


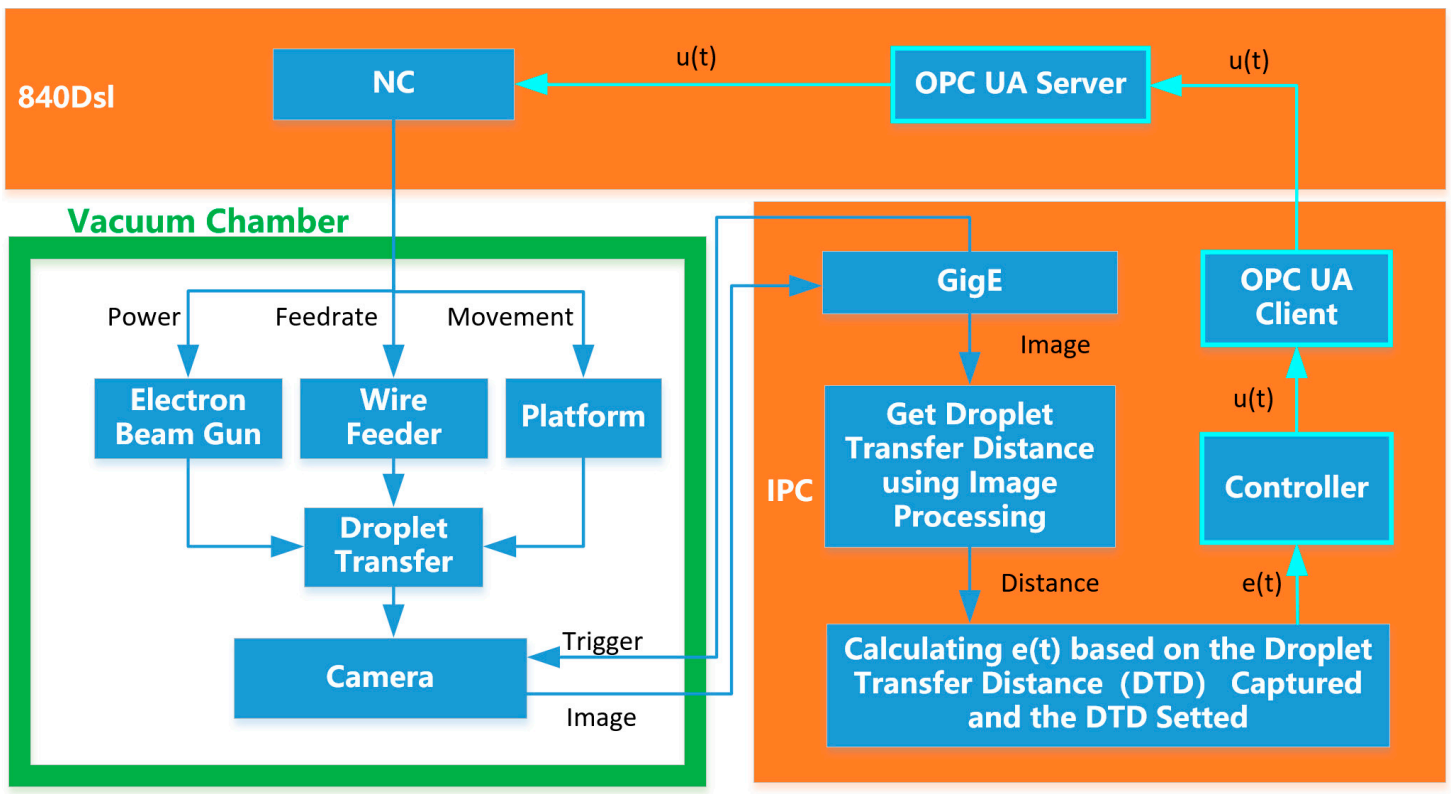

Figure 10. Flow chart of the measurement system.

\section{Results}

\subsection{Detection Accuracy of Droplet Transfer Distance}

For the open-loop experiment described in Table 1, the droplet transfer distance is detected by the image processing algorithm. Figure 11 shows the detected droplet transfer distance versus the actual droplet transfer distance. It can be seen from the figure that the linearity of the detection method is very high and the correlation coefficient reaches up to 0.99 . Figure 12 is the measurement error versus the actual droplet transfer distance. Ninety-five percent of the points fall within $\pm 0.08 \mathrm{~mm}$ around the actual droplet transfer distance. The maximum error is $0.14 \mathrm{~mm}$, which meets the demand of the closed-loop control of droplet transfer.

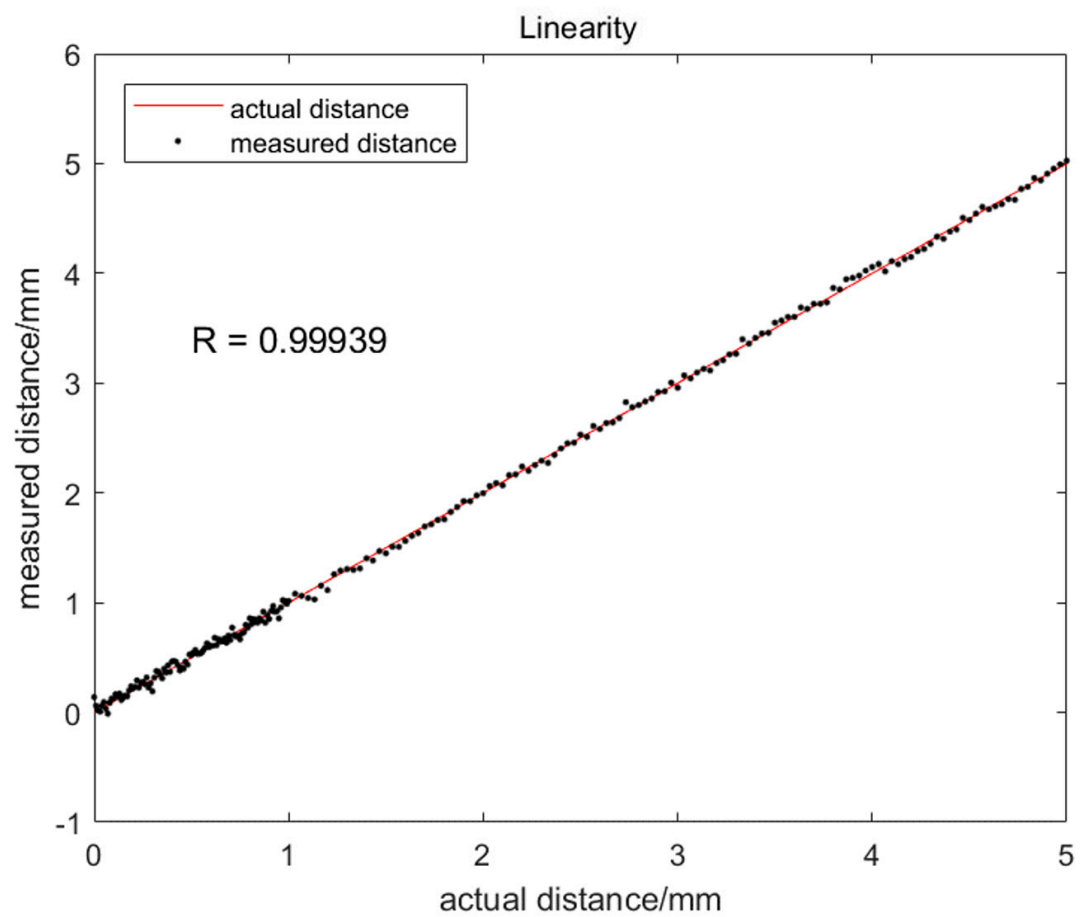

Figure 11. Linearity of Droplet Transfer Distance Detection Method. 


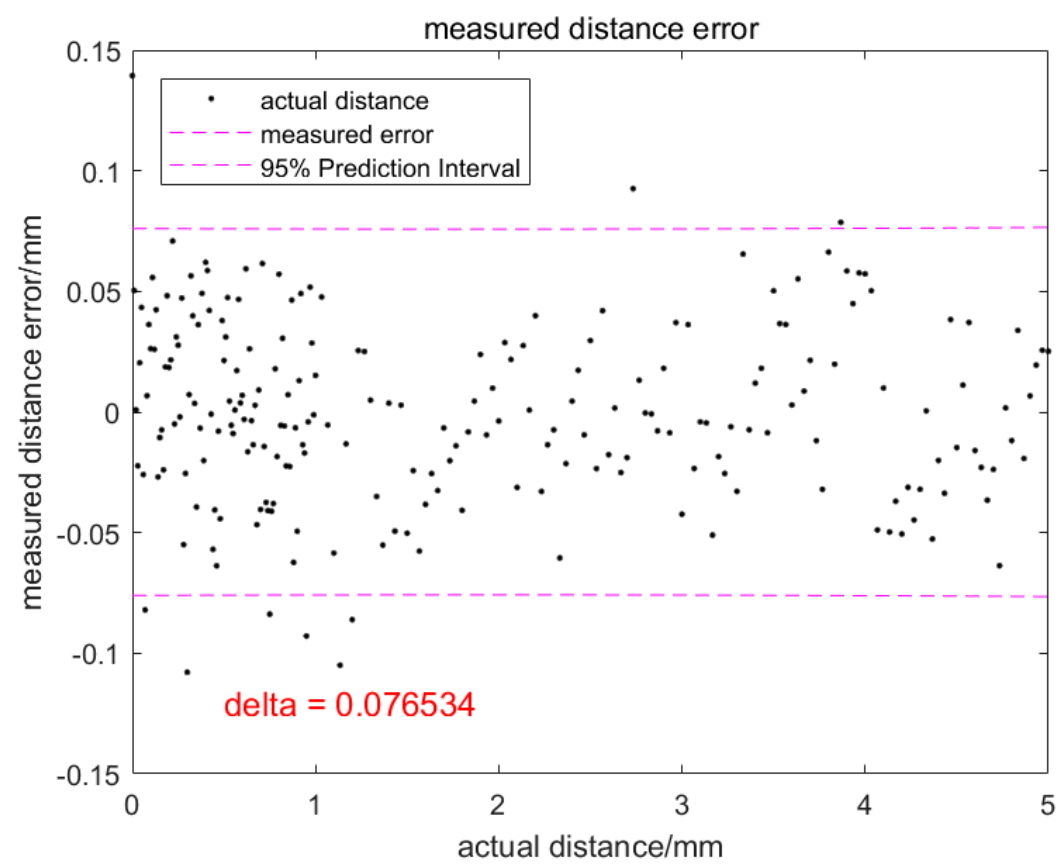

Figure 12. Droplet transfer distance detection error.

\subsection{Closed-Loop Control Accuracy of Droplet Transfer Distance}

The real-time droplet transfer distance within the closed-loop control is shown in Figure 13. It can be seen that the liquid bridge length can be maintained within $\pm 0.2 \mathrm{~mm}$ uniformly and stably.

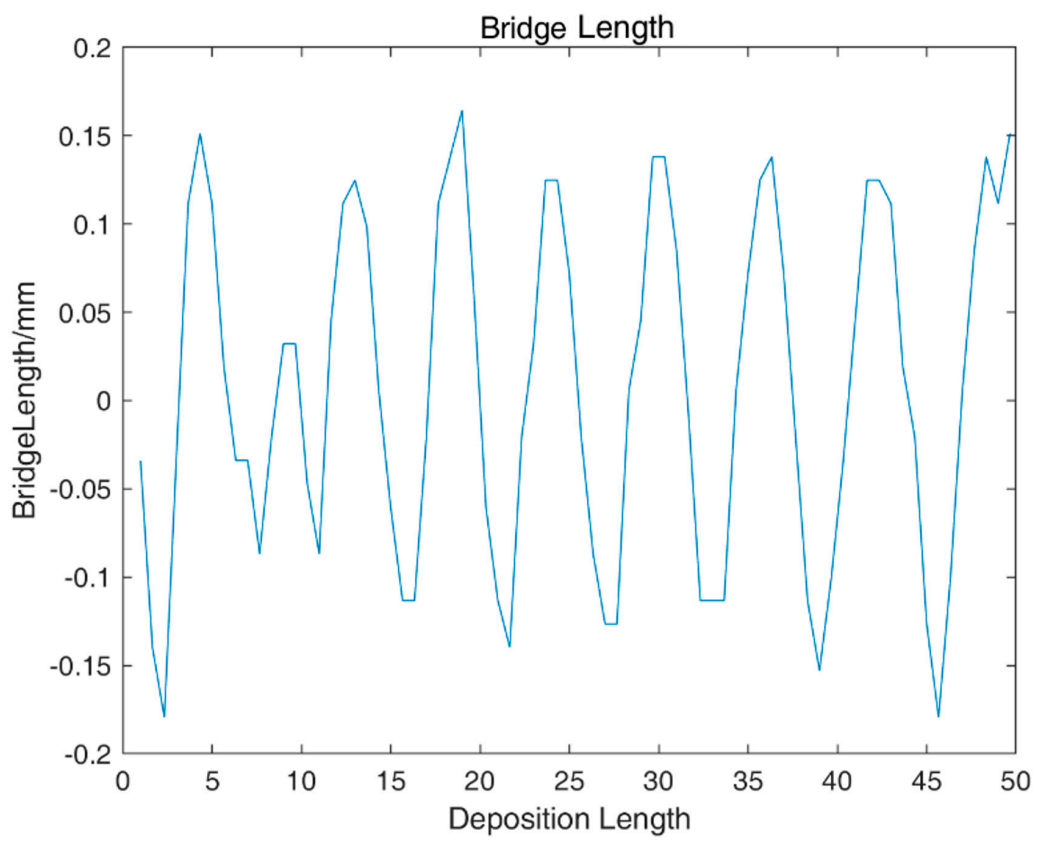

Figure 13. Actual liquid bridge length during closed-loop control of the droplet transfer.

The deposition effect of the open-loop and closed-loop control is shown in Figure 14. It can be seen that, when the droplet transfer distance changes from 0 to $5 \mathrm{~mm}$ without control, the droplet transfer state changes from liquid bridge to large droplet transfer, and the deposition quality is poor. When the droplet transfer state is maintained as a liquid bridge transfer within closed-loop control of the droplet transfer distance, the deposition quality is consistent, which is better than that without control. 


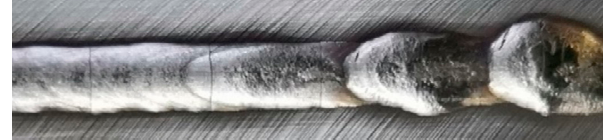

(a)

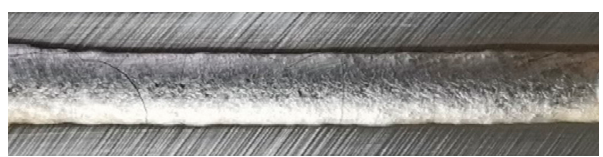

(b)

Figure 14. Deposition result: (a) deposition within the open-loop control; (b) deposition within the closed-loop control.

\section{Conclusions}

In this paper, a droplet transfer detection system is outlined which can clearly photograph the droplet transfer image and the stable liquid bridge process interval $(0 \sim 1 \mathrm{~mm})$ is obtained by different droplet transfer distance experiments. In addition, a method of droplet transfer distance detection is proposed, and the detection accuracy is $\pm 0.1 \mathrm{~mm}$. Moreover, a closed-loop control method for droplet transfer in EBF3 based on image processing is proposed. In the closed-loop control experiment, the droplet transfer can be realized stably. Furthermore, the control accuracy is $\pm 0.2 \mathrm{~mm}$. The deposition quality is effectively improved using this closed-loop control method, which provides a basis for future metal additive manufacturing in space. In addition, a custom-built image processing algorithm is developed in order to improve the detection stability of droplet transfer. The reliability of the algorithm is improved by threshold binarization, local minimum segmentation, and dilatation/erosion.

Author Contributions: Conceptualization, methodology, software, writing-original draft preparation, S.C.; supervision, writing-review and editing, B.C., H.X. and D.D.; formal analysis, investigation, validation, data curation, visualization, H.Z., L.W. and X.S. All authors have read and agreed to the published version of the manuscript.

Funding: This research received no external funding.

Acknowledgments: This work is sponsored by Project 51875309 supported by National Natural Science Foundation of China, the National Key Research and Development Program of China (2017YFB1103100).

Conflicts of Interest: The authors declare no conflict of interest.

\section{References}

1. Herderick, E. Additive manufacturing of metals: A review. In Proceedings of the Materials Science \& Technology 2011 Conference \& Exhibition, Columbus, OH, USA, 16-20 October 2011; pp. 1413-1425.

2. Wong, K.V.; Hernandez, A. A Review of Additive Manufacturing. ISRN Mech. Eng. 2012, $2012,208760$. [CrossRef]

3. Taminger, K.M.; Domack, C.S.; Taminger, B.L.; Hafley, R.A.; Burke, E.R. In-process thermal imaging of the electron beam freeform fabrication process. In Proceedings of the SPIE 9861, Thermosense: Thermal Infrared Applications XXXVIII, Baltimore, MD, USA, 11 May 2016.

4. Taminger, K.M.B.; Hafley, R.A. Electron beam freeform fabrication: A rapid metal deposition process. In Proceedings of the 3rd Annual Automotive Composites Conference, Troy, MI, USA, 9-10 September 2003.

5. Chang, S.; Gach, S.; Senger, A. A new 3D printing method based on non-vacuum electron beam technology. J. Phys. 2018, 1074, 012017.

6. Seufzer, W.J.; Taminger, K.M. Control of Space-Based Electron Beam Free Form Fabrication. 2007. Available online: https://ntrs.nasa.gov/archive/nasa/casi.ntrs.nasa.gov/20070030308.pdf (accessed on 10 January 2020).

7. Taminger, K.M.; Hafley, R.A.; Martin, R.E.; Hofmeister, W.H. Closed-Loop Process Control for Electron Beam Freeform Fabrication and Deposition Processes. U.S. Patent No. 8,452,073, 28 May 2013.

8. Song, L.; Bagavath-Singh, V.; Dutta, B. Control of melt pool temperature and deposition height during direct metal deposition process. Int. J. Adv. Manuf. Technol. 2012, 58, 247-256. [CrossRef]

9. Trapaga, G.; Matthys, E.F.; Valencia, J.J. Fluid flow, heat transfer, and solidification of molten metal droplets impinging on substrates: comparison of numerical and experimental results. Metall. Trans. B 1992, 23, 701-718. [CrossRef] 
10. Heralić, A.; Christiansson, A.K.; Lennartson, B. Height control of laser metal-wire deposition based on iterative learning control and 3D scanning. Opt. Lasers in Eng. 2012, 50, 1230-1241.

11. Wu, B.; Ding, D.; Pan, Z. Effects of heat accumulation on the arc characteristics and metal transfer behavior in Wire Arc Additive Manufacturing of Ti6Al4V. J. Mater. Process. Technol. 2017, 250, 304-312. [CrossRef]

12. Tang, L.; Landers, R.G. Layer-to-layer height control for laser metal deposition process. J. Manuf. Sci. Eng. 2011, 133, 021009. [CrossRef]

13. Hafley, R.; Taminger, K.; Bird, R. Electron Beam Freeform Fabrication in the Space Environment. In Proceedings of the 45th AIAA Aerospace Sciences Meeting and Exhibit, Reno, NV, USA, 8-11 January 2007.

14. Heralić, A.; Christiansson, A.K.; Ottosson, M. Increased stability in laser metal wire deposition through feedback from optical measurements. Opt. Lasers Eng. 2010, 48, 478-485. [CrossRef]

15. Dos Santos, E.; Kuroiwa, L.; Ferreira, A. On the visualization of gas metal arc welding plasma and the relationship between arc length and voltage. Appl. Sci. 2017, 7, 503. [CrossRef]

16. Zeng, J.; Chang, B.; Du, D.; Peng, G.; Chang, S.; Hong, Y.; Wang, L.; Shan, J. A Vision-Aided 3D Path Teaching Method before Narrow Butt Joint Welding. Sensors 2017, 17, 1099. [CrossRef] [PubMed]

17. Zeng, J.; Chang, B.; Du, D.; Hong, Y.; Chang, S.; Zou, Y. A Precise Visual Method for Narrow Butt Detection in Specular Reflection Workpiece Welding. Sensors 2016, 16, 1480. [CrossRef] [PubMed]

18. Zeng, J.; Chang, B.; Du, D.; Wang, L.; Chang, S.; Peng, G.; Wang, W. A Weld Position Recognition Method Based on Directional and Structured Light Information Fusion in Multi-Layer/Multi-Pass Welding. Sensors 2018, 18, 129. [CrossRef] [PubMed]

19. Chang, S.; Zhang, H.; Xu, H.; Hong, Y.; Wang, L.; Du, D. Online Path Recognition for Additive Manufacturing and Arc Welding with Filler Wire Based on Multi-vision Information Fusion. J. Mech. Eng. 2019, 15, 17.

20. Chang, S.; Zhang, H.; Xu, H. Online Measurement of Deposit Surface in Electron Beam Freeform Fabrication. Sensors 2019, 19, 4001. [CrossRef] [PubMed]

(C) 2020 by the authors. Licensee MDPI, Basel, Switzerland. This article is an open access article distributed under the terms and conditions of the Creative Commons Attribution (CC BY) license (http://creativecommons.org/licenses/by/4.0/). 